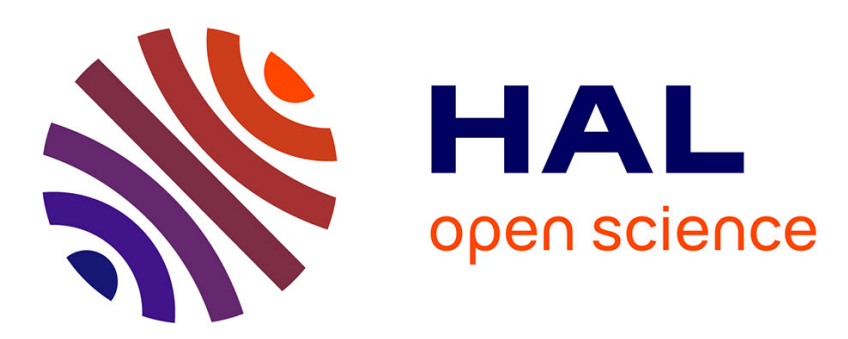

\title{
Enabling Technologies for future learning scenarios: The Semantic Grid for Human Learning
}

Pierluigi Ritrovato, Matteo Gaeta, Francesco Orciuoli, Angelo Gaeta

\section{To cite this version:}

Pierluigi Ritrovato, Matteo Gaeta, Francesco Orciuoli, Angelo Gaeta. Enabling Technologies for future learning scenarios: The Semantic Grid for Human Learning. Proceedings of the IEEE International Symposium on Cluster Computing and the Grid (CCGrid 2005), 2005, Cardiff, United Kingdom. 8 p. hal-00190095

\section{HAL Id: hal-00190095 \\ https://telearn.archives-ouvertes.fr/hal-00190095}

Submitted on 23 Nov 2007

HAL is a multi-disciplinary open access archive for the deposit and dissemination of scientific research documents, whether they are published or not. The documents may come from teaching and research institutions in France or abroad, or from public or private research centers.
L'archive ouverte pluridisciplinaire HAL, est destinée au dépôt et à la diffusion de documents scientifiques de niveau recherche, publiés ou non, émanant des établissements d'enseignement et de recherche français ou étrangers, des laboratoires publics ou privés. 


\section{Enabling Technologies for future learning scenarios: The Semantic Grid for Human Learning}

\author{
Angelo Gaeta, Pierluigi Ritrovato \\ Centre for Research in Pure and \\ Applied Mathematic \\ \{agaeta, ritrovato\}@crmpa.unisa.it
}

\author{
Francesco Orciuoli \\ MoMA Srl \\ \{orciuoli\}@momanet.it
}

\author{
Matteo Gaeta \\ DIIMA - University of Salerno \\ \{gaeta\}@unisa.it
}

\begin{abstract}
In this paper, starting from the limitations and constrains of traditional human learning approaches, we outline new suitable approaches to education and training in future knowledge based society. In our vision, learning and teaching are no longer standalone activities but complex, conversational and experiential-based processes implying collaboration, direct experience, mutual trust and shared interests. We identify characteristics of the environments suitable for these processes, and we compare different enabling technology infrastructures in order to justify why the Semantic Grid for Human Learning, that is a particular enhanced instance of the traditional Semantic Grid, is the most appropriate infrastructure to build our vision on. Finally, we present a realistic learning scenario as a case study, proving the effectiveness of our innovative learning approaches for future Education and Training.
\end{abstract}

\section{Introduction}

For many years, learning approaches have been based on traditional information transfer paradigm in which there is a central figure, the teacher, whose primary activity is the provisioning of educational contents in order to transmit information to learners. These approaches aim at monitoring learner's progresses and to give him a score. Success or failure depends on abilities and capabilities of the teacher, but the better teaching is only a necessary condition for the better learning. In order to provide a wider education, to reduce costs and to overcome constrains due to traditional learning contexts, Information and Communication Technologies (ICT) have been adopted by industries and research centres to develop e-Learning solutions. These solutions are mainly infrastructures for distance learning that adopt the rather narrow pedagogic paradigm of information transfer, allowing the teacher to select particular pieces of information, to prepare educational contents and to make them available to students that can consume the proposed contents when and where they want. In other words, eLearning solutions represent a merely "digitalization" of traditional learning approaches, and this is exactly what they had to do because it was enough in the context of the so-called Information Society. Nevertheless, they present the same issues as the traditional learning approaches, for instance: the adoption of a simple information transfer model, they are content centred, the learner has a passive role, they are context unaware. Nowadays, we are living in an evolving society, in which dynamicity and competitiveness are required and knowledge. rather than information, are a central issue in our life. Education and training are fundamental to prepare young learners to be critical and responsible members of this kind of society, and we realize the necessity of new learning approaches useful for this purpose. The paper is organized as follow: section 2 illustrates the key features of our vision on human learning for Education and Training. Section 3 presents some enabling technologies "semantic-aware" and provides a simple evaluation of their key features that can be useful to build our vision and justifying our choice. Section 4 presents a particular instance of the Semantic Grid, that we believe the most suitable infrastructure for human learning. Section 5 illustrates a case study, followed by our conclusions and future works.

\section{Our vision: learning as a complex experiential-based conversational process}

To improve the human learning, many educational models were proposed by researchers and scientists coming from different fields and a lot of paradigms aroused from these models bringing to the development of many e-Learning solutions that implement a particular model instead of another. Anyway, the current learning solutions handle the human learning as an "activity" and they do not take into account all the features of the human learning. For instance, they do not adequately consider: (a) the importance of the dialogue in human learning; (b) the centrality of the learner with respect to the learning strategies; (c) that human learning cannot be separated from the social context in which it happens; (d) that the main purpose of human learning is to generate an intelligent behaviour, allowing human beings not only to act and react to changes surrounding them (experiential), but also to reflect and acquire knowledge about what is surrounding them. Moreover, to choose a particular educational model, and to implement an e-Learning solution on its basis, means to superimpose to learners

Work partially supported by the European Community under the Information Society Technologies (IST) programme of the 6th Framework Programme for RTD - project ELeGI, contract IST-002205. This document does not represent the opinion of the European Community, and the European Community is not responsible for any use that might be made of data appearing therein 
how they have to learn and to the teachers how they have to teach. This is not a good strategy because it is not absolutely true that a model is better than others and, especially, that one model is able to capture all the feature of the human learning.

On this basis, we propose a more effective approach for human learning and teaching, not based on a particular educational model, but incorporating features of different educational models into a paradigm that focuses on the learners and on the way they create and share knowledge.

Human learning is a process that we define: (i) complex - complexity has different meanings in literature (as explained in [7] and [8]), and it is not our purpose to analyse its meaning. We use the term "complex" to indicate that the learning process consists of many interactions between the learner and other entities belonging to his social context and related to different pedagogical situations. These interactions heavily depend on one another and an interaction can have no predictable influences on the other; (ii) conversational - conversation is a key feature of the human learning process: it implies an active collaboration with other students, teachers, tutors, experts or, in general, other human peers. Conversation, in fact, is more than a simple exchange of opinions or information. Participants have to be established, they have to agree upon some topics, they have dynamic roles and they can join and dynamically resign the conversation; (iii) knowledge based - different levels of knowledge are required in the human learning process. Domain knowledge representations are clearly necessary but they are not enough: knowledge about learner and hi/her social context are important as well. The learner's features cannot be separated from the social context in which the learning process happens and they both dynamically change; (iv) ubiquitous and pervasive not just anytime/anywhere but, more generally, the capability to support multiple diverse pedagogical models and to automatically adapt them. Pervasiveness is another important aspect of human learning that is, by itself, a pervasive process; (v) quality driven - from the learner's perspective, quality is a degree of satisfaction and it depends on the learner's preferences too. Our process has to deal with different kind of qualities from Quality of Collaboration, taking into account dimensions like social cooperation and/or tutoring support, to Quality of (Learning) Experience, emphasizing dimensions like interactivity with educational resources and/or multimedia enhancement.

The essence of our approach is to create dynamic contexts that provide adequate conditions for each learner (taking into account his/her features and social context, providing tutoring and enhanced presence), that support him/her during his/her learning process (also through the use of technologies for collaboration, highly realistic virtual scientific experiments, real time simulations, personalized learning path definitions, stateful message exchange sessions to support conversation) and, finally, that let him/her free to reason about what is useful to achieve his/her goal creating, in this way, the knowledge model suitable for him/her. In our vision, the role of the teacher is no more overemphasized, he/she comes into this process as a domain expert whose purpose is to propose educational goals and topic of discussion, to moderate discussions inside a community, to give feedbacks to learners and to prepare educational contents.

\section{Enabling technologies for next generation learning environments: is there a winner?}

As stated above, to support the human learning process we need to create for each learner a dynamic and social context centred on him/her. This context can be seen as an instance of a particular and advanced learning environment, providing many features for learners, like the ones described in [5] and offering: (i) support for autonomous and dynamic creation of communities. The learner should be able to search and find peers having similar skills and sharing the same learning objectives, he /she should be able to organize groups with clear educational goals and to collaborate with participants to achieve these goals. In this active collaboration, peers in the group should be supported by tutors or more expert learners; (ii) support for active and realistic experiments. Learning resources should be interactive, engaging, and responsive. Furthermore, real world input, such as live stock market prices or remote sensing data, should be easy to integrate; (iii) support for personalization. The learner is the central figure upon which we have to create a dynamic context: skills, preferences and needs of the learner as well as the knowledge about his/her social context should be take into account in the discovery of resources and services, the selection of target concepts to learn and the preparation of educational contents; (iv) support for knowledge creation and evolution. The learners should be free to create, adopt and propose their own knowledge model. As in the real world, knowledge evolves over the time and it is distributed among different people and communities, which adopt their most appropriate knowledge model. The knowledge creation should be also supported by the use of data and text mining, feature extractions tools, advanced tools for semantic tagging.

Making real what we have described above is not a simple task, while it is crucial an enabling infrastructure 
with several stringent technical requirements that we are analysing in brief. First, support to communities implies the resources and services sharing, the dynamic negotiation of different kinds of "community agreements" (e.g. pedagogical goals of a community, policies on community resources, roles of community participants), the information and knowledge management tools. Collaboration activities inside a community need stateful message exchange sessions, communication tools, and adoption of interactive resources for active and realistic experiments. Realism requires certain degree of quality in provisioning of data, services and application and this raise quality issues from a technological viewpoint. Selfadaptability of the infrastructure is required to support personalization, and stateful sessions have to be managed in order to restart a learning session where the learner logged off last time. Pervasiveness should be supported from both the viewpoints of an easy integration of multiple different types of devices, including wearable and mobile devices, and of creation of educational scenarios in which the learner is immersed in all the aspects of a learning process that also takes care of cultural and social contexts.

Standards as well as technologies are cornerstones of an enabling infrastructure and international efforts, like the ones of IMS Global Learning Consortium [14] or Advanced Distributed Learning [15], they are essential to achieve genuine interoperability and meaningful interactions in open distributed systems.

Realizing the need of enabling technologies in our vision of the human learning and that currently more than one with many of the features described above is available, we try to analyze three semantic-aware technologies in order to choose the one that, according to us, is the most suitable to build our human learning process upon.

\subsection{Agent}

An agent defines a software system characterised by the following properties [11]: (i) autonomy: agents encapsulate some state, and make decisions about what to do based on this state; (ii) reactivity: agents are situated in an environment, they are able to perceive this environment, and able to respond in a timely fashion to changes that occur in it; (iii) pro-activeness: agents do not simply act in response to their environment, they are able to exhibit goal-directed behaviours by taking the initiative; (iv) social ability: agents interact each other via some kind of agent-communication language and typically have the ability to engage in social activities in order to achieve their goals. Agents are also classified in literature with respect to their features. In our context the most useful "kind" of agents, are the Pedagogical Agents defined by Johnson [16] as "autonomous agents that support human learning, by interacting with students in the context of interactive learning environments. ... They adapt their behaviour to the dynamic state of the learning environment, taking advantage of learning opportunities as they arise. They can support collaborative learning as well as individualized learning, because multiple students and agents can interact in a shared environment".

Agent systems typically provide their effectiveness when two o more agents interact or work together to satisfy a goal in a Multi Agent System (MAS) [17].

\subsection{Semantic Web}

What is the Semantic Web? According to BernersLee, Hendler, and Lassila [3] "The Semantic Web is an extension of the current web in which information is given well-defined meaning, better enabling computers and people to work in cooperation". The basic idea to fulfil this ambitious dream is to enable advanced automatic processing of web contents so that data can be shared and processed by both humans and software in a decentralized environment. Current efforts are related to the definition and implementation of standards and technologies for the Semantic Web, such as RDF [18], RDF Schema [19], OWL [10]. The efforts are now moving toward definitions of Rules languages and Logic framework as well as tools for Annotation, Collaboration and Automated Knowledge Access [20]. Beyond the importance of ontologies and tools for the Semantic Web infrastructure, it is critical the development of applications [3]. As any other promising vision, the Semantic Web has given rise to a lot of expectations and, sometimes, misunderstandings. What is clear is that the Semantic Web will be a powerful infrastructure that will enhance the Web, allowing users to be helped to perform their task by agents and services and, probably, it will change the way the Web is now perceived. Currently the Web is one "big thing" that users use for different and mainly "standalone" purposes, like common searches. The creation of some type of communities or groups is also possible by using collaboration tools, but the lack of semantics makes this kind poor of collaboration. The Semantic Web has the capability to split this big thing into many parts and to personalize any part for a single user. In this way, any user will perceive its "own web" contextualized for his/her purpose, in which he/she has his knowledge, his/her peers to collaborate with, his/her personal contents, agent and services helping him/her during his/her tasks. In other words, the current Web will be a web of Semantic Webs each of them providing a contextualized environment for users. 


\subsection{Semantic Grid}

The Semantic Web is to Web as the Semantic Grid is to Grid: the key factor is always the semantics. In the beginning, Grid was synonym of meta computing and according to this first vision, Kesselman and Foster attempted a first definition of the Grid [1]: “...a hardware and software infrastructure that provides dependable, consistent, pervasive, and inexpensive access to high-end computational capabilities". Research has brought to a new vision of Grid that became synonym of infrastructure to manage "coordinate resource sharing and problem solving in dynamic, multi-institutional virtual organizations" where the focus is on the concept of Virtual Organization (VO) [21]. Currently, the evolution of Grid is marked by the adoption of a service oriented approach in designing Grid architectures. The Open Grid Services Architecture (OGSA) integrates key Grid technologies with Web Services mechanisms to create a distributed system framework for integrating, virtualizing and managing resources and services within distributed, heterogeneous and dynamic VO [2]. Upon the OGSA model different kinds of Grids have been raised like Computation, Data or Information Grid. Apart from the specific term, the key features of all these Grids are virtualization in term of services and dynamic policybased provision of what is virtualized. Some of them deal with well known issues, like Computational Grid that provides the access to a large virtual computer and Information Grid permitting the access to a large virtual information source.

While these Grids are well known, more or less, most recent terms may cause confusion: what does Cognitive Grid mean, for example? A discussion about Cognitive, Knowledge and Semantic Grids is provided in [22], in which it is emphasized that "In the end all these terms deal with adding intelligence to the Grid" and that "The basis for understanding all three terms is the Semantic Web". This was clearly understood by De Roure and other researchers that by analogy with the Semantic Web define the Semantic Grid [13] [23] as: "an extension of the current Grid in which information and services are given well-defined meaning, better enabling computers and people to work in cooperation". The Grid is improved through standards and technologies of the Semantic Web community, to make explicit and machine understandable the knowledge about resources and services of a Grid infrastructure, communities and individuals of communities. As for the Semantic Web, the semantics will allow automatic negotiation, discovery and composition of services but even in this case, to fulfil the "e-Research" vision [13], agents or brokers, helping researchers to perform their tasks, are mandatory.

\subsection{What can they do for our vision of learning?}

To develop a learning environment upon distributed MAS infrastructures, we have to build a set of pedagogical agents that interact with learners, each of them incorporating one o more pedagogical capabilities and having a pedagogical goal. For instance, a pedagogical agent can subsume the role of teacher providing customisation of didactic experiences on the basis of student's preferences and target objectives retrieved from a student's model. As any other agents, the pedagogical ones are autonomous and context-aware, the notion of virtual community is supported and they also support very well, dynamic and flexible interactions involving negotiation, discovery and cooperation. The Agent's features are very useful for the purpose of human learning and they fit very well in a service-orientedmodel.

The notion of agent is present both in the Semantic Web and in the Semantic Grid. The Semantic Web is born with the agent in mind and, with a minor emphasise, this is true also for the Semantic Grid. A recent research effort of Grid community is related to study how models and theories of agent computing can be reused in the context of the Grid one. As recognized by Foster et al. in [6] and De Roure et al. in [13], some ideas of the agent's world can be adopted in the Grid's one, for example autonomy and dynamic negotiation of agent can help research fields related to self-management and SLA negotiation and management of the OGSA model. At the same time, the reverse is true: agents can benefit from the powerful Grid infrastructures. On the basis of these reciprocal synergies between the service oriented model and agent one, we can suppose that both the Semantic Web and the Semantic Grid will became "agent-like". But the question still stands: who will provide better benefits to human learning between the Semantic Web and the Semantic Grid? Theoretically, both the Semantic Web and the Semantic Grid are able to provide dynamic and social contexts, with the features emphasized in the section opening, supporting our human learning process. They both support personalization and knowledge creation, acquisition and evolution very well, and can provide a wide and heterogeneous set of services and didactical resources for active experiences.

Support to autonomous and dynamic creation of communities is clear for Grid technologies, as they are key technologies enabling the VO paradigm. In a VO, all the participants have the same goal and collaborate and share resources and services in order to achieve the common goal that, in our context, could be the knowledge acquisition through active experiences. This statement that is true for the Grid in general, is also true for the 
Semantic Grid but it is not so clear for the Semantic Web. Actually, already in [3] Berners-Lee et al. they clarify that the Semantic Web "is not "merely" the tool for conducting individual tasks ... if properly designed, the Semantic Web can assist the evolution of human knowledge as a whole" and introduce the notion of community. In addition, Stutt and Motta in [9] define the concept of Knowledge Neighbourhoods as "locations in cyberspace where learners can congregate into groups or larger communities with the goal of acquiring knowledge about some topic" that is, more or less, the same concept of dynamic VO. What is not so clear is how to "properly" design the Semantic Web to allow creation of something like the Knowledge Neighbourhoods. From this viewpoint, we think Grid technologies are more mature, but we do not believe it is a discriminating factor in order to prefer Grid technologies instead of the Semantic Web ones. In conclusion, we do not see a winner and we cannot say that one infrastructure is clearly better than the other for our purpose. We are also aware that the full accomplishment of the Semantic Grid vision requires at least a partial achievement of the Semantic Web one.

Even if the future is hard to predict, we have decided to move our research's effort towards the Semantic Grid for two distinctive features that we argue useful for fulfilment of our human learning vision.

The first one is the underlying service model. Conversation is the key for our learning process and it must be supported with a stateful and dynamic service model. While the Semantic Web is neutral with respect to the underlying service model (it doesn't suggest nor prevent this kind of model), the Semantic Grid relies upon interfaces and behaviour defined by the Web Services Resource Framework (WSRF) [24] that defines the WS-Resource as "stateless service acting upon a stateful resource" and proposes a service model of an intermediate complexity between pure stateless services and pure conversational ones, thus allowing a simple way to compose services without loosing the advantages of state management. The second one is the improvement on the scale of computational power and data storage that the Semantic Grid has regarding to the Semantic Web. This improvement is needed if we wish to support realistic experiments involving responsive resources, 3d simulations, immersive VR.

\section{Enhancing the Semantic Grid: the Semantic Grid for Human Learning}

As stated above, we believe that the Semantic Grid will be a good enabling infrastructure for creation of learning environments allowing our human learning process. Obviously, the Semantic Grid is for e-Research in general and it should be enhanced with right arms in order to be useful for a particular field of e-Research, like Education \& Training. We define the Semantic Grid for Human Learning as a domain verticalization of the Semantic Grid improved with tools, services, standards and technologies for the Education \& Training. In our human learning process we envisage the centrality of the learner with respect to the learning strategies. Having this purpose in mind, we will not customize the Semantic Grid for a specific pedagogical model. Instead, we have to catch all the pedagogical features we have identified including collaboration, personalization, contextawareness, realism, ubiquity, accessibility and availability.

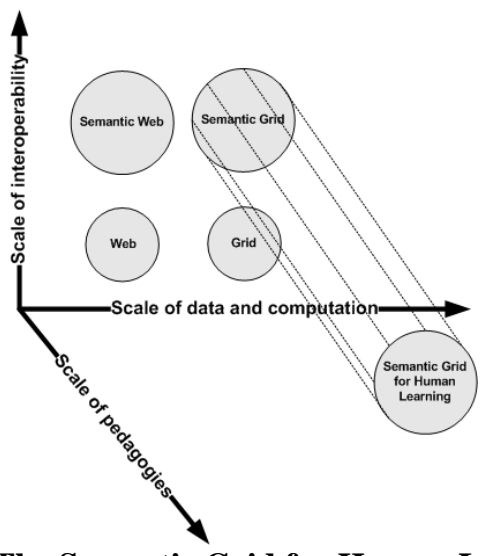

Figure 1 - The Semantic Grid for Human Learning Vision

To clarify our vision, we can refer to the well known two axis diagram that places the Semantic Grid into a two dimensional spaces, the scale of interoperability and the scale of data/computation. The Semantic Grid for Human Learning can be seen as an expansion of the Semantic Grid on a third axis measuring the supported scale of pedagogies. To create this particular instance of the Semantic Grid, we foresee: (i) adoption of IMS Learning Design (IMS-LD) specifications - IMS LD [4] that is a specification used to describe learning scenarios. It can describe a wide variety of pedagogical models, including group work and collaborative learning. It does not define individual pedagogical models; instead, it provides a high-level language, or meta-model, that can describe many different models. The language describes how people perform activities using resources and services, and how to coordinate them into a learning flow; (ii) adoption of the Web Services for Remote Portlets (WSRP) standard - WSRP [25] simplifies the integration of remote contents into portals allowing users to select from a rich choice of contents and integrate them in their portal to obtain an easy personalization. The integration of 
WSRP and WSRF in the Grid Middleware Fabric layer allows the creation of an underlying services model that is dynamic, stateful and presentation-oriented. WSRP, in fact, defines presentation-oriented services in contrast to the data-oriented ones. While data-oriented services are web services that receive requests and return data objects encoded in XML documents in the response, the WSRP services include presentation and optionally interactions as a part of the service itself.

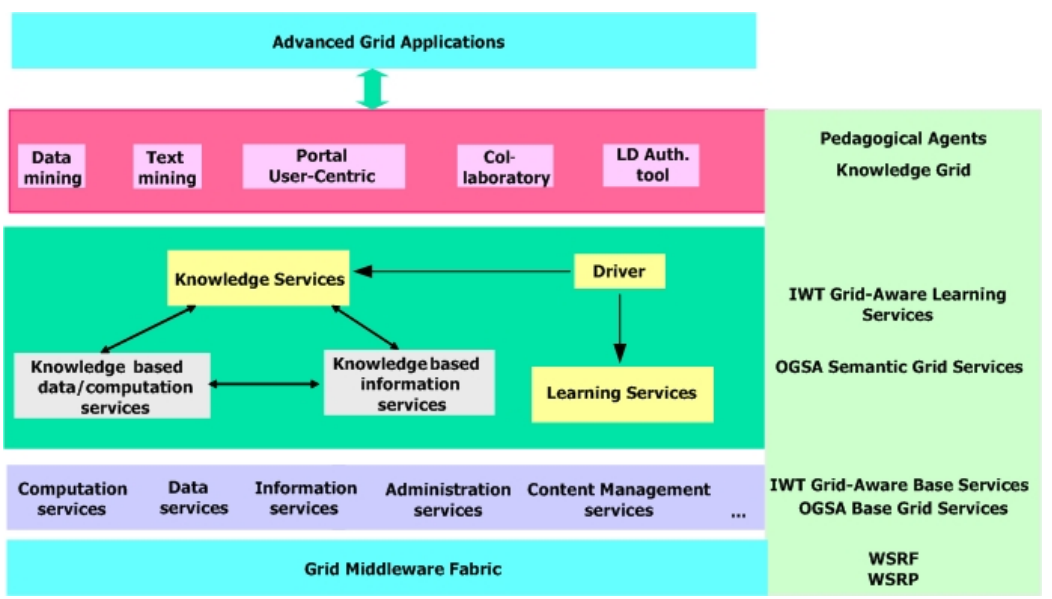

Figure 2 - The Semantic Grid for Human Learning Architecture service against its description to find the service that best satisfy the requirements.

From an architectural viewpoint, the Semantic Grid for Human Learning is shown in the Figure 2, based on the reference architecture due to Globe et. al. [23]. In the Semantic Grid for Human Learning, we foresee pedagogical agents to support learners during their learning experiences. The pedagogical agents can rely upon a set of semantically enriched services typical of the learning domain that we have identified in our previous works [12] and we collectively define as IWT Grid-Aware Services. IWT Grid-Aware [12] is a current effort of the CRMPA whose aim is the reengineering of a commercial product named IWT (Intelligent Web Teacher) using Grid technologies. IWT [29] is an application framework that facilitates the creation of advanced learning solutions filling up the lack of support for flexibility and extensibility in existing elearning systems. In IWT Grid-Aware, two sets of services are identified: the Base Services providing functionalities typical of a Learning Management System and the Learning Services providing high-level functionalities for a personalized learning experience, like learning path personalization, student 's model evaluation and Driver services for management and delivery of learning experiences.

Learning experiences are created by the teacher using IMS-LD authoring tools that produce a LD Document describing a learning scenario. In order to execute the LD Document, we have to bind each activity with a set of resources and services, which in IMS terminology is called "environment", able to execute the activity. Knowledge services can be used to bind the learner's preferences and the pedagogical model against tools, resources and services available on the Grid. After this binding phase, there is the delivery of the learning experience to the learner. Delivery is provided by particular interactive services called Drivers that deliver the learning experiences taking into account the learner's preferences on the presentation and visualization, thus generating the right portlets that the user-centric portal, acting as a WSRP Consumer, is able to integrate and aggregate. To support the interactions among the actors involved in a scenario, trusted collaboration groups can be dynamically created where learners and teachers can join and resign the scenario. These collaboration groups are created when in a IMS-LD play there are acts containing shared activities among actors with different roles. 


\section{A Learning Scenario addressing course personalization in a Grid environment}

GUI. The service will respond with a SOAP message in which it will put a frame of XHTML code. The engine, acting a WSRP Consumer, will extract the XHTML code

The scenario we are going to show concerns a learning approach that foresees a course personalization based on learner's cognitive state and preferences that are obtained accessing learner's profile stored in a knowledge base and dispensing pre-assessment tests. The roles in the scenario are learner, human tutor and Intelligent Tutoring System (ITS) able to update learner's profile in order to reflect the knowledge acquired by learner and to build personalized remedial works, which are a short courses containing didactic material explaining the concepts that are not well understood by learners in

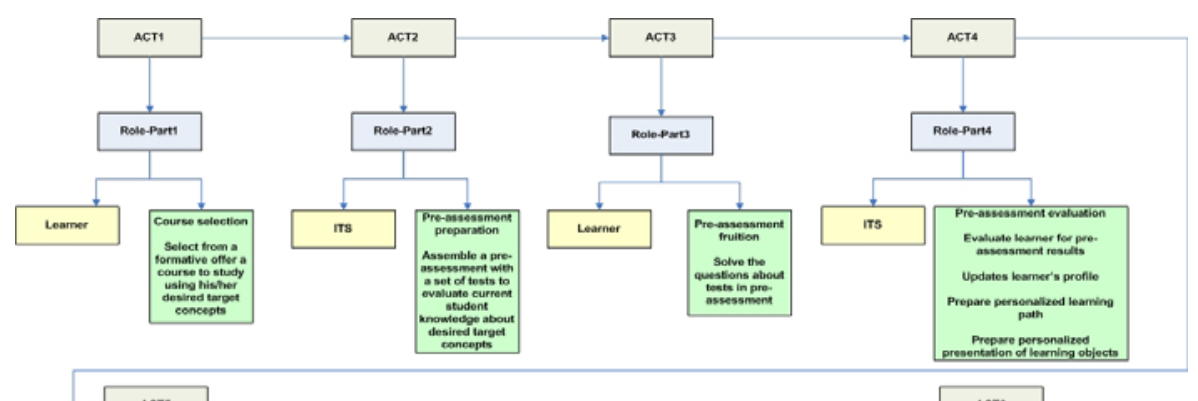
the previous didactic course frame. Using the formalism of IMS LD, we obtain the method depicted in Figure 3, in which each activity of an act (for instance, the activity Course Selection of the Act1) needs a particular environment, which is a set of services and/or resources, to be executed. To execute the above method, we need a IMS LD engine. Each act needs appropriate resources and services. The first are contents by which learners acquire knowledge and the services are functionalities invoked during the learning process in order to communicate with tutors or other learners. Currently, both resources and services have to be statically bound to the LD scenario, but this is not a feasible approach to our scenario. In fact, the didactic course presentation have to be assembled in a customised way by ITS that links specific learning objects to the presentation using learner's cognitive state and preferences. Therefore, different learners with different profiles have to obtain different didactic course presentations for the same learning scenario execution.

In order to allow a personalized course preparation and presentation, we need: (i) to build services that are able to provide an operative environment in which users can interact with a GUI; (ii) to allow a dynamic binding of resources and services distributed in a Grid environment based on learner's features. The first problem is addressed through the adoption of the underlying service-model defined in the context of the Semantic Grid for Human Learning. When the IMS LD engine has to render the GUI for a specific activity in a learning scenario, it will invoke the service asking for a

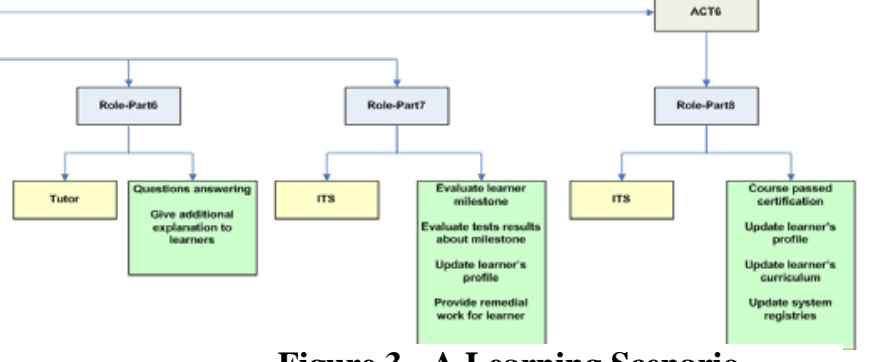

Figure 3 - A Learning Scenario

from the message and compose a new web page, to be aggregated in the User-Centric portal, that graphically implements the activity in the learning scenario by which the user can perform his/her interactions. For the resolution of the second problem, we introduce an abstraction level between learning scenario and resources, in order to implement the binding with contents at runtime, introducing services providing the delivery of learning objects. The decision about the choice of resources to present to a specific learner is taken by "Prepare learning object presentation service", and the decision algorithm is based on the information extracted from learner's profile (cognitive state and preferences) using "Learner's profile service". These services are implemented in the context of IWT Grid-Aware and through their adoption, we can eliminate the constraint of design-time binding between activities and resources. Anyway, the aforementioned services have to be localized inside the Grid environment. In order to solve this issue, we have to provide a mechanism ensuring a dynamic binding for services. Inside a LD scenario the references to services will be replaced by syntactic and semantic specifications of the service we need. At the executiontime, when the engine has to invoke the service, it will interact with a "Locator" service, that reasoning on the semantically enriched specifications is able to localize, inside the Grid environment, the required service.

The described scenario is only one among the possible scenarios that benefit from our approach. In fact, the 
proposed scenario adopts a collaborative and personalized approach but, dynamicity and adaptability of Semantic Grid for Human Learning architecture, allows us to use the same scenario for an experiential-based approach for which we have only to bind more interactive resources that provide adequate GUI in the course presentation, based on WSRP. Furthermore, our architecture also allows mapping different pedagogical methods in different LD scenarios that can be indexed and reused as any other resource of the VO. All LD scenarios, even if based on different pedagogical methods, can use services and tools defined in our architecture.

\section{Conclusions and future works}

In this paper, we have presented our vision of human learning for future Education \& Training. We have also introduced the Semantic Grid for Human Learning that is a particular enhanced instance of the traditional Semantic Grid providing useful features to support our vision, from the technological point of view, pointing out what are the motivations that, in our opinion, bring us to invest in the Grid technologies. The Semantic Grid is a general vision for e-Research, and in this paper we have discussed of how to customize this vision for the Education \& Training. We hope that similar efforts related to other fields of e-Research may arise in order to bridge the powerful general vision of the Semantic Grid with practical requirements of many e-Research communities. Currently, at the CRMPA we have an application framework that facilitates the creation of advanced learning solutions filling up the lack of support for flexibility and extensibility in existing e-learning systems and we are reengineering this framework to be GridAware. As future works, we are planning to develop highlevel pedagogical agents that can take full advantages from the services developed in the context of IWT GridAware and can be used to build complex human learning scenarios.

\section{Bibliography}

[1]. Foster I. and Kesselman C.: The Grid: Blueprint for a New Computing Infrastructure, Morgan Kaufmann, 1999

[2]. Foster I., Kesselman C., Nick J. and Tuecke S.: The Physiology of the Grid: An Open Grid Service Architecture for Distributed System Integration, 2002

[3]. Berners-Lee T., Hendler J. and Lassila O.: The Semantic Web, Scientific American, May 2001

[4]. http://www.imsglobal.org/learningdesign/index.cfm

[5]. Ritrovato P., Gaeta M., Cerri S. A. and Alison C.: Services, Semantics and Standards for Future Learning Scenarios. Proc. of the GLS Workshop at ITS 2004 Maceio, Brazil.
[6]. Foster I., Jennings N.R. and Kesselman C.: Brain Meets Brawn: Why Grid and Agents Need Each Others, 2004

[7]. Axelrod, Robert and Michael Cohen: Harnessing Complexity, The Free Press, New York, 1999

[8]. Gell-Mann, Murry: What is Complexity?, 1995

[9]. Stutt A. and Motta E.: Semantic Learning Webs. Journal of Interactive Media in Education. March, 2004

[10].McGuinness D. and van Harmelen F: OWL Web Ontology Language Overview, W3C Recommendation, Febr. 2004

[11].Wooldridge, M. and Jennings N.R.: Intelligent agents: Theory and practice. The Knowledge Engineering Review, 10(2):115-152, 1995

[12]. Capuano N., Gaeta A., Laria G., Orciuoli F and Ritrovato P.: How To Use GRID Technology for Building Next Generation Learning Environments. To appear in the book Towards the Learning GRID: advances in Human Learning Services. Publisher: IOS Press in the series: "Frontiers in Artificial Intelligence and Applications".

[13].De Roure D., Jennings N. R. and Shadbolt N.R.: The Semantic Grid: Past, Present and Future, will be appearing in the March 2005 issue of IEEE Proc.

[14].http://www.imsproject.org/

[15].http://www.adlnet.org/

[16].Johnson, W.L.: Pedagogical agents. Proc. of ICCE '98, the Sixth Intern. Conference on Computers in Education, Beijing, China: 14-17 October.

[17].V. R. Lesser: Cooperative Multiagent Systems: A Personal View of the State of the Art. IEEE Trans. on Knowledge and Data Engineering, Vol 11 (1), p. 133-142, 1999

[18].Manola F. and Miller E.: RDF Primer, W3C Recomm., Febr., 2004

[19].Guha R.V. and Brickley D.: RDF Vocabulary Description Language 1.0: RDF Schema, W3C Recomm., Febr., 2004,

[20].Semantic Web Activity: Advanced Development, http://www.w3.org/2000/01/sw/

[21].Foster I., Kesselman C. and Tuecke S.: The Anatomy of the Grid: Enabling Scalable Virtual Organizations. Intern. Journal of Supercomputer Applications, vol. 15, 2001

[22].Geldof M.: The Semantic Grid: will Semantic Web and Grid go hand in hand?, report for the Grid technologies unit of the European Commission, June 2004

[23].Goble C. and De Roure D.: The Semantic Grid: Myth Busting and Bridge Building. Proc. of the 16th European Conference on Artificial Intelligence, Valencia, 2004

[24].Foster I, Frey J., Tuecke S. et al.: The WS-Resource Framework, 2004

[25].Schaeck T. and Thompson R.: Web Services for Remote Portlets (WSRP) Whitepaper, 28 may 2003.

[26].Allison C. and Michaelson R.: Design Consideration for an ELeGI Portal. Proc. of 3rd Intern. LeGE-WG Workshop. Berlin, Germany. 3 December, 2003

[27].http://www.elegi.org/index.php

[28]. The OWL Service Coalition: OWL-S: Semantic Markup for Web Services, 2003.

[29].Capuano N., Gaeta M., Micarelli A.and Sanginetto E.: An Intelligent Web Tutoring System for Learning Personalization and Semantic Web Compatibility. Proc. of PEG 2003, St. Petersburg, Russia. 